\title{
Therapeutic Complement Targeting in ANCA-Associated Vasculitides and Thrombotic Microangiopathy
}

\author{
Pavel Novikov ${ }^{a} \quad$ Natalia Kozlovskaya ${ }^{a}$ Sergey Moiseev ${ }^{a} \quad E^{\prime}$ guene Shilov ${ }^{a}$ \\ Irina Bobkova $^{a}$ Adrian Schreiber $^{b, c}$ Dmitry Tsvetkov ${ }^{b, c}$ Maik Gollasch $^{\text {b-d }}$ \\ Nancy Mahe ${ }^{\mathrm{e}}$ Khadija El Amranie ${ }^{\mathrm{e}}$ Andreas Kurtz \\ a.M. Sechenov First Moscow State Medical University, Moscow, Russia; ${ }^{b}$ Experimental and \\ Clinical Research Center, a Joint Cooperation between the Charité Medical Faculty and the \\ Max Delbrück Center for Molecular Medicine at the Charité and the Max Delbrück Center for \\ Molecular Medicine in the Helmholtz Association, ' Nephrology and Intensive Care Medicine, \\ Campus Virchow, and ${ }^{d}$ Koch Metchnikoff Forum, Section Nephrology, and ${ }^{\mathrm{e}}$ Berlin-Brandenburg \\ Center for Regenerative Therapies (BCRT), Charité - Universitätsmedizin Berlin, Berlin, Germany
}

\begin{abstract}
What Is It about?
Anti-neutrophil cytoplasmic antibody (ANCA)-associated vasculitides (AAVs) are systemic autoimmune diseases of medium-to-small vessels. Recent findings predominantly from animal studies showed a crucial role of the complement system in the pathogenesis of AAVs, which had previously been demonstrated only for thrombotic microangiopathy (TMA). Following a multidisciplinary approach, we argue that therapies directed against the complement system point to the necessity of a genetic workup of genes of complement components and regulators in patients with AAV and TMA. We conclude that genetic testing together with pluripotent stem cells and bioinformatics tools may broaden our approach to the treatment of patients with aggressive forms of AAVs.
\end{abstract}

\section{Keywords}

ANCA-associated vasculitis · C5b-9 - Opsonins · CCX168 $\cdot$ C5a receptor · Human pluripotent stem cells · Genetic testing · Complement

\section{Abstract}

Anti-neutrophil cytoplasmic antibody (ANCA)-associated vasculitides (AAVs) are a group of systemic autoimmune disorders characterized by necrotizing inflammation of medium-tosmall vessels, a relative paucity of immune deposits, and an association with detectable circulating ANCAs. AAVs include granulomatosis with polyangiitis (renamed from Wegener's granulomatosis), microscopic polyangiitis, and eosinophilic granulomatosis with polyangiitis 
(Churg-Strauss syndrome). Until recently, AAVs have not been viewed as complement-mediated disorders. However, recent findings predominantly from animal studies demonstrated a crucial role of the complement system in the pathogenesis of AAVs. Complement activation or defects in its regulation have been described in an increasing number of acquired or genetically driven forms of thrombotic microangiopathy. Coinciding with this expanding spectrum of complement-mediated diseases, the question arises as to which AAV patients might benefit from a complement-targeted therapy. Therapies directed against the complement system point to the necessity of a genetic workup of genes of complement components and regulators in patients with AAV. Genetic testing together with pluripotent stem cells and bioinformatics tools may broaden our approach to the treatment of patients with aggressive forms of AAV.

\section{Introduction}

Anti-neutrophil cytoplasmic antibody (ANCA)-associated vasculitides (AAVs) are systemic disorders with characteristic features of necrotizing inflammation of small and medium vessels. AAVs comprise several clinically and pathologically defined disease entities: (1) granulomatosis with polyangiitis (renamed from Wegener's granulomatosis), mainly associated with ANCA against proteinase 3 (PR3); (2) microscopic polyangiitis, mainly associated with ANCA against myeloperoxidase (MPO); (3) eosinophilic granulomatosis with polyangiitis (renamed from Churg-Strauss syndrome); and (4) renal-limited pauci-immune necrotizing and crescentic glomerulonephritis [1]. AAVs are primarily caused by leukocyte migration and subsequent tissue damage. Although it is clear that genetic and environmental factors are involved, the pathophysiological mechanisms behind AAVs are still not fully understood despite decades of research.

\section{The Complement System in Experimental AAV}

ANCAs are directed against the granule proteins MPO or PR3, and there is evidence from animal and in vitro studies that ANCAs are pathogenic and cause effector functions in neutrophils and monocytes, leading to subsequent tissue damage [2,3]. Binding of ANCA IgG to membrane-expressed ANCA antigens on the surface of preactivated neutrophils (primed) leads to full activation of these cells, resulting in degranulation of tissue-damaging proteases, generation of reactive oxygen species, generation of inflammatory cytokines such as IL-1 $\beta$, and generation of neutrophil extracellular traps.

Until recently, AAVs have not been viewed as a complement-mediated disease. However, increasing data predominantly from animal studies have demonstrated a crucial role of the complement system in the pathogenesis of AAV. The first animal study using a passive anti-MPO antibody transfer model showed that mice deficient in either complement factor B or complement component C5 were protected from disease, whereas complement component C4-deficient mice developed disease comparable to wild-type mice. These results suggested a central role for the alternative pathway of complement activation, whereas the classic or lectin-binding pathway is apparently not involved [4]. In a different study, anti-MPO necrotizing and crescentic glomerulonephritis could be blocked by treating mice with a C5-inhibiting monoclonal antibody [5]. However, it is still unclear as to which tissue-damaging mechanism of the complement system is involved in the pathogenesis of AAV. 


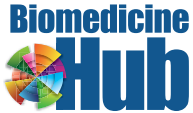

Fig. 1. Complement in anti-neutrophil cytoplasmic antibody (ANCA)-associated vasculitis. I. Cytokine-primed neutrophil granulocytes are activated by binding of ANCA IgG to surfaceexpressed ANCA antigens. II. This process leads to full activation, resulting in the release of ROS and proteases. III. The alternative pathway of complement activation is activated, leading to generation of the $\mathrm{C} 5$-convertase complex (C3b Bb P). IV. The potent anaphylatoxin $\mathrm{C} 5 \mathrm{a}$ is generated, which binds to its receptor, $\mathrm{C} 5 \mathrm{aR}$ (V.) expressed on the neutrophil surface, leading to further neutrophil activation as an amplification loop.
Biomed Hub 2016;1:453106 (DOI: 10.1159/000453106)

Novikov et al.: Therapeutic Complement Targeting in ANCA-Associated Vasculitides and Thrombotic Microangiopathy

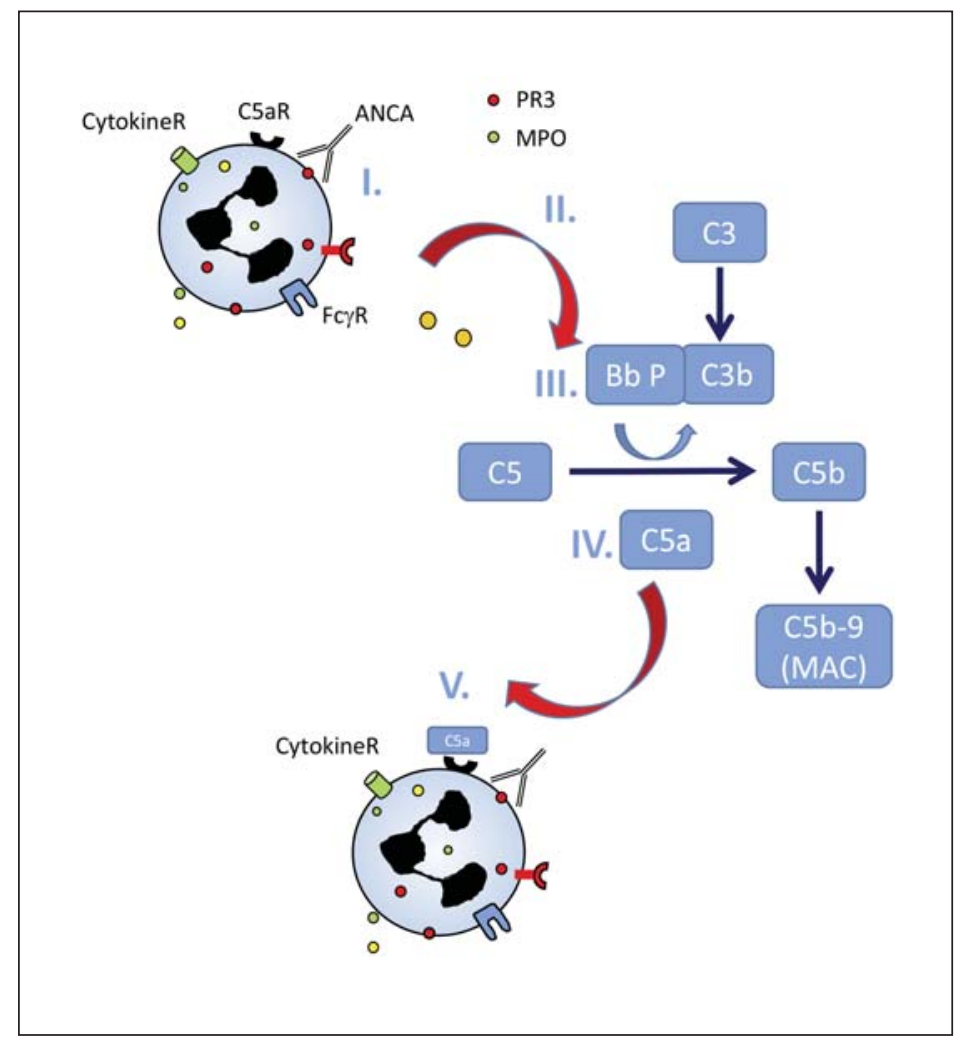

In general, the complement system could potentially mediate AAV disease via 3 different mechanisms: (1) a direct tissue-damaging effect by generation of C5b-9 (the terminal membrane attack complex [MAC]); (2) generation of opsonins (C3b, iC3b, and C3d), which decorate target surfaces through covalent surface binding to favor removal of target structures; or (3) generation of anaphylatoxins ( $\mathrm{C} 3 \mathrm{a}$ and $\mathrm{C} 5 \mathrm{a}$ ), which are potent proinflammatory molecules that attract and activate leukocytes through interaction with their receptors $\mathrm{C} 3 \mathrm{aR}$ and C5aR. In a follow-up study, our group tested the hypothesis that the complement system mediates its action in AAV by generation of anaphylatoxins; in a murine anti-MPO animal model, C5aR-deficient mice were almost completely protected from anti-MPO-induced disease [6]. Furthermore, we could demonstrate that ANCA-stimulated neutrophils release unidentified substances that activate the complement system with generation of C5a (Fig. 1). Recently, this work was confirmed in transgenic mice expressing human C5aR, where a specific small-molecule blocker of human C5aR (CCX168) reduced disease in an anti-MPO model [7]. It is conceivable that C5a, generated with activation of the complement system, binds to its cognate $\mathrm{C} 5 \mathrm{aR}$ expressed on the neutrophil's surface, which in turn preactivates ("primes") the neutrophil for further ANCA IgG activation.

Together, these studies imply an important role of the complement system in the pathogenesis of AAVs. At first glance this is surprising, since complement activation or defects in its regulation have been identified and well appreciated in an increasing number of acquired or genetically driven forms of thrombotic microangiopathy (TMA), which exhibit clinical and histopathological features distinct from those of AAV. However, a subgroup of patients with AAV displays histologic signs of TMA, which is associated with low C3 levels and poor renal prognosis [8]. This could further support the idea of a significant overlap between the AAV and TMA disease entities. 


\section{Complement in Human TMAs}

Complement-mediated atypical hemolytic uremic syndrome (aHUS) results from dysregulation of endothelium-anchored complement activation. This activation results in endothelial damage and platelet activation, which occurs in approximately $90 \%$ of cases of aHUS [9]. The discovery of ADAMTS13, a zinc-containing metalloprotease enzyme that cleaves von Willebrand factor, has permitted a more accurate diagnosis of thrombotic thrombocytopenic purpura (TTP) and an appreciation of overlapping clinical features of TTP and aHUS [9]. While ADAMTS13 deficiency causes TTP, partial ADAMTS13 deficiency is a common finding in aHUS patients, which warrants genetic screening and functional tests of ADAMTS13 in aHUS patients [10].

The alternate complement pathway is continuously active, albeit at a low level. Disturbed function or deficiency of complement regulatory proteins of the alternate pathway leads to continuous generation of C3 and C5 convertases, resulting in formation of the MAC (Fig. 1). The MAC induces lysis of endothelial cells or damage at the site of the endothelium and platelets, which results in TMA in certain vascular beds that are more prone to this injury than others [11-14]. Mutations in genes encoding for complement factor $\mathrm{H}(\mathrm{CFH})$, complement factor I (CF), complement factor B (CFB), membrane cofactor protein $(M C P) / C D 46$, and $C 3$ comprise about $50 \%$ of the known mutations in patients with aHUS $[13,15]$. Another $10 \%$ of aHUS is due to autoantibodies to complement factor $\mathrm{H}$. In about $10-12 \%$ of patients with aHUS, combined mutations of $C F H, C F I, M C P, C 3, C F B$, or thrombomodulin (THBD) may exist [13].

In general, patients with aHUS have a poor prognosis. Thromboses can occur almost everywhere, but smaller blood vessels in the kidneys, lungs, brain, gastrointestinal system, or heart are often involved. More than $60 \%$ of patients with mutations of $C F H, C F I, C 3, C F B$, and $T H B D$ either die or develop end-stage renal disease within 1 year of presentation [16]. Patients with $M C P$ mutations have a better long-term outcome, with only $30 \%$ progressing to endstage renal disease, though they tend to have a disease course with frequent relapses [17].

Plasma exchange therapy is the first-line therapy for children with aHUS. Although plasma infusion replenishes deficient complement regulators, plasma exchange has the added benefit of removing mutant complement factors and/or autoantibodies $[13,18]$. The rate of remission after plasma exchange is variable, depending on the genetic mutations [13]. Plasma exchange is of little benefit to patients with an MCP mutation, as MCP is a transmembrane glycoprotein and no circulating factor [13]. Immunosuppressive agents are of no benefit in aHUS, except to patients with CFH autoantibodies [13]. Eculizumab is a monoclonal antibody to $\mathrm{C} 5$ that prevents cleavage of $\mathrm{C} 5$ to $\mathrm{C} 5 \mathrm{~b}$, thus preventing formation of the MAC (Fig. 1). Eculizumab has been used in patients with aHUS with favorable results [19, 20]. There is a high recurrence rate of aHUS after renal transplantation, which is dependent on the genetic mutation. There is an increased risk of aHUS recurrence in patients with mutations of $C F H, C F I$, and $C 3$, whereas the risk is lower for patients with $M C P$ mutations [13]. Of note, there is an increased risk for donors of developing aHUS, as they might have disease-causing mutations with incomplete penetrance [13]. Thus, if living related kidney transplantation is the only option available to a family, then the donor and the recipient should undergo a thorough genetic evaluation of the complement system, and the donor should be counseled that even if the genetic test is negative, the risk of developing aHUS is not completely eliminated [13].

Thus, genetic screening for aHUS susceptibility factors is well appreciated to help clarify whether a TMA present in a complement-amplifying condition arises from unmasking a genetically driven aHUS, versus a time-limited complement storm-mediated aHUS due to direct endothelial damage in which no genetic predisposition is present [9]. Therefore, a precise molecular diagnosis may help in treating patients with TMA. Clinical use of massively parallel DNA sequencing will provide a way to identify genetic variants in the complement system to better diagnose patients. Although exciting, these advancements are not without 
limitations. Massively parallel sequencing (next-generation sequencing) platforms provide vast quantities of data, but the associated error rates (approx. $0.1-15 \%$ ) are higher - and the read lengths are generally shorter (35-700 bp for short-read approaches) - than those of traditional Sanger sequencing platforms, requiring careful examination of the results and possibly combined approaches with both technologies, particularly for variant discovery and clinical applications [21,22].

\section{The Complement System in Human AAV}

\section{Complement Activation in Human AAV}

Chen et al. [23] investigated the signs of complement activation in renal biopsy specimens of 7 patients with MPO-ANCA-associated pauci-immune glomerulonephritis. The MAC, C3d, factor B, and factor P could be detected in glomeruli and small blood vessels of patients with AAV, while they were absent or scarce in patients with minimal change disease and normal renal tissue. Systemic activation of complement by the alternative pathway was also found in 120 patients with AAV who were distributed into 2 groups based on the presence or absence of active inflammation. Plasma levels of $\mathrm{C} 3 \mathrm{a}, \mathrm{C} 5 \mathrm{a}$, soluble $\mathrm{C} 5 \mathrm{~b}-9$, and $\mathrm{Bb}$ were significantly higher in active AAV than in remission of vasculitis, while plasma levels of properdin were significantly lower in patients in the active stage of disease [24]. Moreover, deposition of $\mathrm{Bb}$ - an alternative complement pathway marker - in glomeruli correlated with the proportion of total crescents, the extent of interstitial infiltrate, interstitial fibrosis, and tubular atrophy. Urinary levels of Bb, C3a, C5a, and soluble C5b-9 were significantly higher in active AAV than in the stage of remission. Urinary levels of $\mathrm{Bb}$ in patients with active AAV correlated with serum creatinine levels [24]. In a recent study, C3d, C4d, and C5b-9 staining was found to be positive in a majority of renal biopsies analyzed. C3d and properdin staining was associated with cellular crescents [25].

These findings provide additional evidence that complement activation through the alternative pathway contributes to the development of AAV (Fig. 2). C5a plays a major role, as it shows strong chemoattraction to neutrophils and is able to prime those cells, thus enabling their full activation by ANCA [4].

\section{Genetic Susceptibility to Human AAV}

To improve the understanding of genetic factors involved in human AAVs, genetic components have been investigated in candidate association studies and 2 genome-wide association studies (GWAS). The majority of the identified genetic AAV risk factors are common variants (Fig. 2). The genes include CTLA4, PTPN22, COL11A2, SERPINA1, and the major histocompatibility complex class II gene cluster [26-29].

A recent meta-analysis determined genetic variants most likely associated with AAV. The study also investigated whether diagnostic and serological subtypes within AAV have distinct genetic backgrounds [30]. The authors identified 33 genetic variants associated with AAV. These genetic variants were in or near the following genes: CD226, CTLA4, FCGR2A, HLA-B, HLA-DP, HLA-DQ, HLA-DR, HSD17B8, IRF5, PTPN22, RING1/RXRB, RXRB, STAT4, SERPINA1, and TLR9. The authors found that in $76 \%$ of the genetic variants, a subdivision based on ANCA serotype resulted in higher odds ratios than a subdivision based on clinical diagnosis. The results indicated that a subdivision of AAV based on ANCA serotype has a stronger genetic basis than a subdivision based on clinical diagnosis [30]. It is important to note that GWAS identify specific DNA locations, not complete genes. Many variants identified in GWAS are nowhere near genes encoding proteins. It is intriguing to speculate that some of the locations could affect complement regulatory mechanisms. In support, Persson et al. [31] found that 


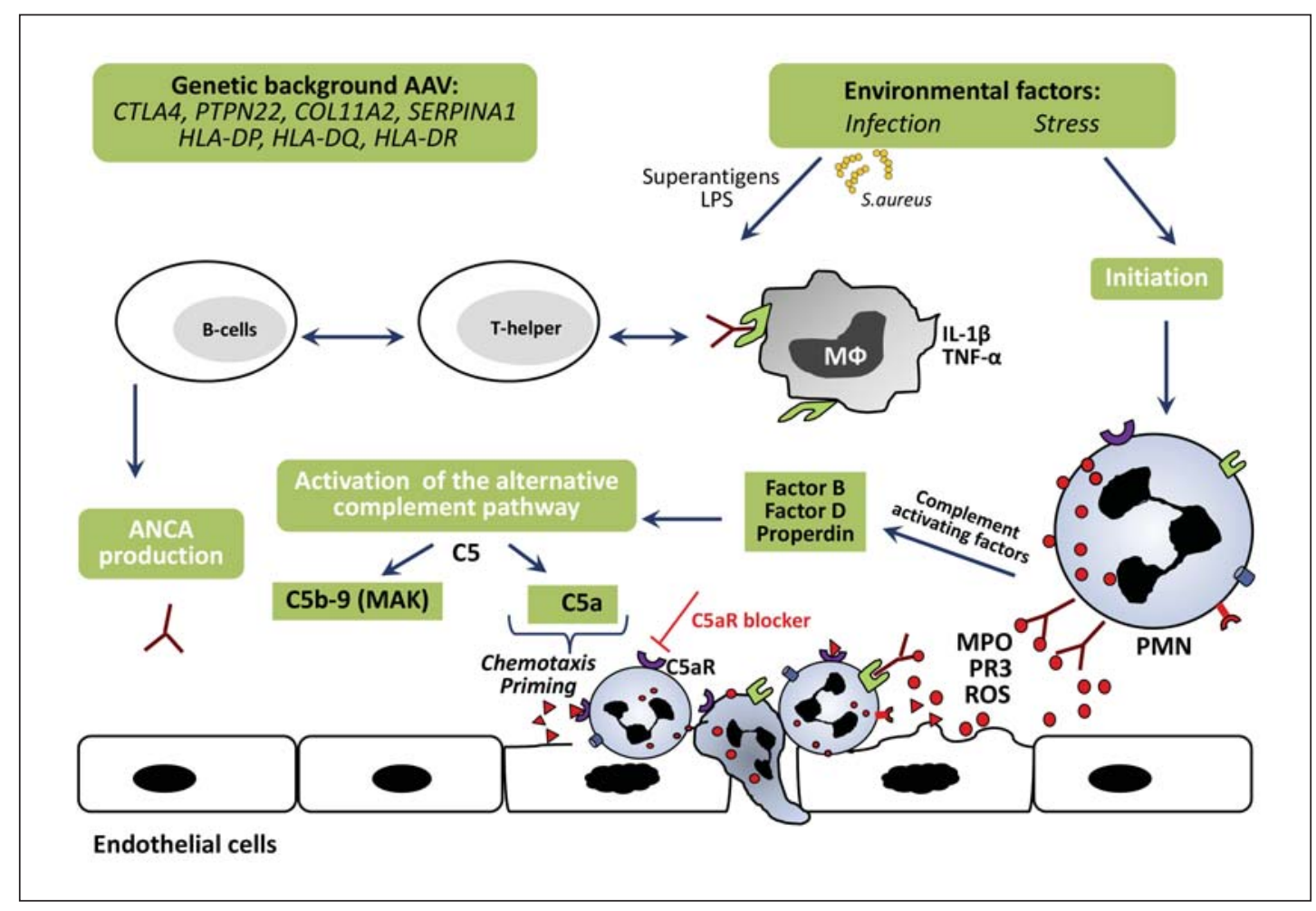

Fig. 2. Pathogenesis of anti-neutrophil cytoplasmic antibody (ANCA)-associated vasculitides (AAVs). In a (genetically) susceptible individual who is exposed to an environmental trigger, such as Staphylococcus aureus, antigen-presenting cells (APCs) result in the proliferation of T-helper (TH; TH-17) cells. These activated $\mathrm{T}$ cells activate macrophages and attract neutrophils. Macrophages release the proinflammatory cytokines IL-1 $\beta$ and tumor necrosis factor- $\alpha$ (TNF- $\alpha$ ), both of which prime neutrophils to display myeloperoxidase (MPO) and proteinase 3 (PR3). APCs process and present these antigens to TH cells, and chronically activated TH cells form effector memory T cells, which then form granulomata. TH cells activate B cells that release ANCAs of the immunoglobulin G type into the bloodstream. In addition, the number of regulatory T cells is likely reduced and their function impaired, allowing for persistent or enhanced B-cell activation. Circulating primed neutrophils become activated by ANCAs following binding to both Fc $\gamma$ and Fab2. Degranulation of bound neutrophils leads to further neutrophil recruitment to the site and to release of reactive oxygen species (ROS) and neutrophil extracellular traps, leading to endothelial cell damage and inflammation.

the allotype frequency of C4A3 among patients with ANCA-positive small vessel vasculitis was increased, and that there was an increase in the C3F allele in patients with PR3-ANCAnegative but not MPO AAV in a British cohort. The authors suggested that genetic factors and the complement system may be of importance in development of the disease, and that these results should increase the interest in studying the complement system in ANCA-associated small vessel vasculitis.

Together, the results point to the necessity of a complete genetic workup of genes of complement components and regulators in patients with AAV and to the importance of a functional characterization of potential pathogenic variants.

\section{Complement as a Target for Treatment in Human AAV}

The oral blocker of the C5aR CCX168 was evaluated in a phase II multicenter clinical trial on AAV patients (NCT02222155). The CLEAR study was designed to assess whether high- 
dose chronic steroids used in the standard-of-care (SOC) regimen for AAV could be reduced or eliminated - without compromising efficacy - by replacement with CCX168. The CLEAR trial met its primary endpoint based on Birmingham Vasculitis Activity Score (BVAS) response at week 12: the proportion of patients with a BVAS response at week 12 was numerically superior and statistically noninferior among patients receiving CCX168 plus low-dose steroids and no steroids as compared to the high-dose steroid-treated SOC group $(86,81$, and $70 \%$, respectively). The mean percent changes in BVAS from baseline to week 12 were 79, 73, and $57 \%$ in the 3 groups, respectively. A higher proportion of patients receiving CCX168 had early clinical remission at week 4 , sustained through week 12 . At 4 weeks, there was a $47 \%$ decrease in albuminuria in patients receiving CCX168 without steroids ( $p=0.0006 \mathrm{vs.} \mathrm{SOC)}$ ) and a $40 \%$ decrease in patients receiving CCX168 plus low-dose steroids ( $p=0.003$ vs. SOC), but an increase of $15 \%$ in patients from the control groups. At 12 weeks, the change from baseline was $-56,-44$, and $-21 \%$ in the 3 groups, respectively. Patients receiving CCX168 (especially without steroids) showed a significant improvement in health-related quality of life domains such as physical functioning, mental health, emotional well-being, pain, and vitality. Estimated glomerular filtration rates improved similarly with CCX168 compared to controls, indicating that steroids are not needed to improve renal function when patients are taking CCX168. CCX168 appeared to be well tolerated by patients with AAV (http://ir.chemocentryx. com/releasedetail.cfm?ReleaseID = 972372). The North American CLASSIC trial is evaluating the safety of 10 or $30 \mathrm{mg}$ of CCX168 twice daily for 12 weeks when added to the full-dose corticosteroid SOC regimen plus cyclophosphamide or rituximab. The CLASSIC safety study met its objectives. The addition of CCX168 to the current SOC therapy did not add safety concerns beyond those seen with SOC alone. The incidence of serious adverse events was similar across the treatment groups in the study (http://ir.chemocentryx.com/releasedetail. cfm?ReleaseID $=976002$ ). ChemoCentryx plans to advance CCX168 into phase III development for AAV.

Taken together there are reliable experimental animal data and data from human marker studies to support the notion that the complement system plays a pathogenic role in AAV. However, further studies are needed to understand exactly how the complement system is activated.

\section{Innovative Approaches to Disease Modeling and Outcome Prediction}

\section{General Considerations}

Human induced pluripotent stem cells (hiPSCs) are donor-specific cell lines which have the capacity to differentiate in all cell types of the human body. They are excellent candidates for individualized disease modeling, as well as for applications in precision medicine. The cells provide the starting material for advanced therapeutic products for clinical use in tissue repair and regeneration. Here, we present an outlook on the use of iPSCs for the modeling of renal diseases and the derivation of cell types from iPSCs which could be applicable in kidney repair. The use of bioinformatics tools and cell-related databases to understand the disease on the cellular level will be described as well.

\section{PSCs for Modeling and Repair of the Kidney}

The kidney is a complex organ with at least 26 different cell types and an intricate anatomical structure necessary to facilitate regulation of homeostasis, electrolyte concentrations, the extracellular fluid volume, and blood pressure. After birth, the human kidney has almost no ability to regenerate. Repair is limited to cases of acute kidney injury, but whether repair occurs is not predictable; moreover, the endogenous capacity for repair of acute 


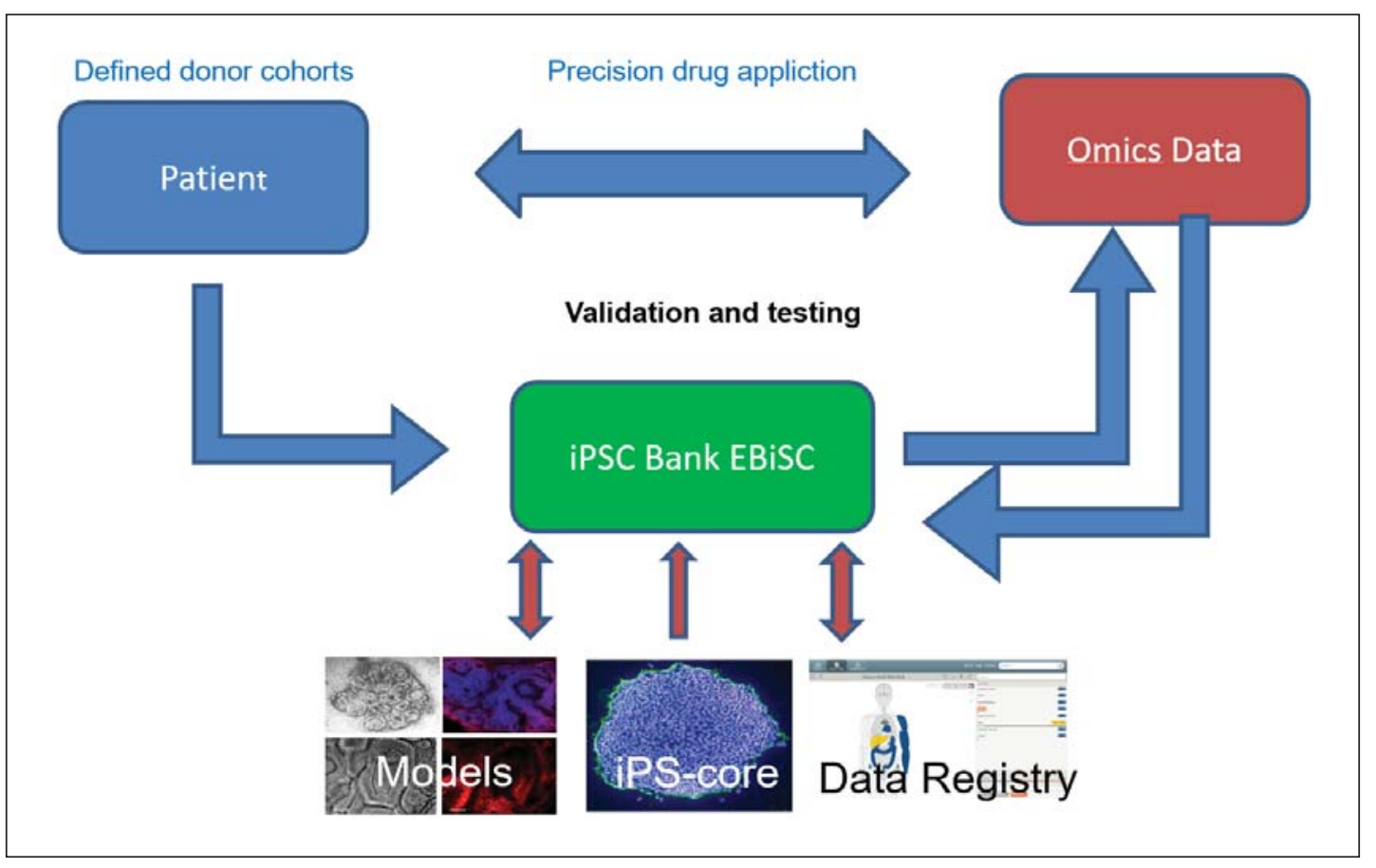

Fig. 3. Human induced pluripotent stem cells (iPSCs) will be derived from defined cohorts of patients who have been characterized by omics technologies and for whom, for example, genotype-phenotype/disease associations have been found. There is a clear need to validate the functional implications of this association for safe translation into precision medicine. Human iPSC banks will be established from these cohorts to provide a widely usable resource of patient surrogates for these validation studies, which can be used not only for renal diseases. The human iPSCs will be generated in a dedicated iPSC core facility. For the kidney, we established the basis for diverse model systems by deriving renal organoids and renal cell types from human iPSCs. In addition, we use and are developing bioinformatics tools and databanks to assess cell- and tissuerelated data for their comparability with the iPSC-derived cell lines and model systems.

damage declines over time. The interplay of the different cell types in chronic and acute disease progression in human patients is not completely understood, and hence the role of different cell types during degeneration and repair remains unclear.

hPSCs are able to differentiate into all cell types of the human body. With the development of reprogramming technology [32], it became possible to derive hiPSCs from any person. This created the opportunity to derive any renal cell type from human donors. Since hiPSCs are genetically identical to the donors, they provide a basis for modeling kidney disease on an individual level and for cell-based therapies. At the Berlin-Brandenburg Center for Regenerative Therapies (BCRT), we were able to differentiate hiPSCs into the major precursor cell types of the human metanephros: metanephric mesenchyme and ureteric bud epithelial cells. Moreover, we could differentiate these cells further into different mature renal cell types, including podocytes, mesangial cells, proximal and distal tubular epithelial cells, and collecting duct epithelial cells. In addition, we were able to derive human kidney organoids at the renal vesicle stage, providing a 3-dimensional model of kidney organogenesis and the kidney (Fig. 3). These findings allow us now to investigate the applicability of the different cell types to cell-based therapies of chronic and acute kidney disease [33]. In addition, the option of differentiating different renal cell types from iPSCs allows us to analyze disease phenotypes not only on the cellular level but also on an individual basis. In addition, interactions of different renal cell 
types - or between renal cells and hiPSC-derived cells of other organs such as hepatocytes or variants of the complement system - can now be assessed in vitro on a patient-specific level. Furthermore, by genetically modifying the cells, it is possible to elucidate the effect of genes and specific mutations on disease progression or on drug responsiveness.

It is thus possible to verify in vitro the association between a patient's drug responses or biomarker composition and variations found on the "omics" level (e.g., of genetic polymorphisms) or pathogenic variations (Fig. 3). These possibilities justify the establishment of hiPSC banks to cover sufficiently large cohorts of patients with renal disease as a resource for disease modeling. These models are then applied to verify omics-disease phenotype associations, and to test in vitro the associated pathways, including interactions with the complement system. We currently use the technology in order to establish hiPSC lines from patients with focal segmental glomerulosclerosis, a devastating progressive kidney disease, to analyze patientspecific phenotypes in hiPSC-derived renal organoids and renal cell types. In addition, we use hiPSC-derived renal cells in microfluidic chips to model their response to drug treatments. Furthermore, the effect of circulating biomarkers from kidney disease patients on the different personalized cell types could be analyzed in such a microfluidics-based perfused system. In the other direction, biomarkers secreted by the model cells could easily be analyzed in this perfused microfluidics model and directly associated with cell type-specific pathological changes.

\section{Bioinformatics Tools}

It is currently very difficult to obtain data on the cell type and single-cell levels, which are needed to characterize a cell and, importantly, to assess changes occurring during development and pathogenesis. Such depth of characterization is also necessary to unambiguously classify hiPSC-derived renal cell types by comparing these cells to their native equivalents. Technological tools are now available to characterize cells on the single-cell level and to analyze the associated data [34]. At the BCRT, we have developed a registry of cell type-associated data as a first step to characterize most or all distinct cell types of the kidney and other organs (Fig. 3). Analytical tools have been developed to compare and classify cells and tissues based on their gene expression profiles. Furthermore, we developed a tool for identifying tissue- and cell-specific marker genes based on their expression patterns [35]. Since cell typespecific reference data are rarely available, especially for native cells of the kidney, we aim at generating these data by single-cell RNA sequencing from healthy and diseased tissues.

In summary, we set up a pipeline starting with generating hiPSCs and differentiating these into multiple renal cell types as well as generating 3-dimensional renal organoids in vitro. These are being used for disease modeling of focal segmental glomerulosclerosis and other renal diseases with the goal to identify the basis of their pathogenic mechanisms and to screen for drugs that counter these mechanisms. These approaches are substantiated by bioinformatics tools for cell analysis and by comparison on the molecular and functional levels. The cell type-specific data will be used to establish a virtual atlas of the kidney which ranges from the molecular to the subanatomical and tissue levels. Finally, we are developing in vitro test systems to elucidate regenerative applications of hiPSC-derived cells, and to analyze drugs regarding their regenerative and repair potency in kidney models. Eventually, we will apply validated cells with a clear mode of action to patients in advanced therapy settings.

\section{Conclusions}

Recent data predominantly obtained from animal studies demonstrated a crucial role of the complement system in the pathogenesis of AAV. In coincidence with this expanding spectrum of complement-mediated diseases, the question arises as to which AAV patients 
might benefit from complement-targeted therapy. We believe that therapies directed against the complement system point to the necessity of a genetic workup of genes of complement components and regulators in patients with AAV. Genetic testing, PSCs and bioinformatics tools will provide new insights into the pathophysiology of AAVs and may broaden our approach to the treatment of patients with aggressive forms of AAV.

\section{Acknowledgments}

The work of the authors is supported by the Koch Metchnikoff Forum, the Deutsche Forschungsgemeinschaft (DFG; A.S. and M.G.), the Deutsche Akademische Austauschdienst (DAAD; M.G.), and the Dr. Werner Jackstädt-Stiftung (M.G.).

\section{Disclosure Statement}

The authors declare no conflicts of interest.

\section{References}

1 Jennette JC, Falk RJ, Bacon PA, Basu N, Cid MC, Ferrario F, Flores-Suarez LF, Gross WL, Guillevin L, Hagen EC, Hoffman GS, Jayne DR, Kallenberg CG, Lamprecht P, Langford CA, Luqmani RA, Mahr AD, Matteson EL, Merkel PA, Ozen S, Pusey CD, Rasmussen N, Rees AJ, Scott DG, Specks U, Stone JH, Takahashi K, Watts RA: 2012 revised International Chapel Hill Consensus Conference Nomenclature of Vasculitides. Arthritis Rheum 2013;65: $1-11$.

2 Falk RJ, Jennette JC: ANCA are pathogenic - oh yes they are! J Am Soc Nephrol 2002;13:1977-1979.

-3 Jennette JC, Falk RJ, Gasim AH: Pathogenesis of antineutrophil cytoplasmic autoantibody vasculitis. Curr Opin Nephrol Hypertens 2011;20:263-270.

-4 Xiao H, Schreiber A, Heeringa P, Falk RJ, Jennette JC: Alternative complement pathway in the pathogenesis of disease mediated by anti-neutrophil cytoplasmic autoantibodies. Am J Pathol 2007;170:52-64.

-5 Huugen D, van Esch A, Xiao H, Peutz-Kootstra CJ, Buurman WA, Tervaert JW, Jennette JC, Heeringa P: Inhibition of complement factor C5 protects against anti-myeloperoxidase antibody-mediated glomerulonephritis in mice. Kidney Int 2007;71:646-654.

6 Schreiber A, Xiao H, Jennette JC, Schneider W, Luft FC, Kettritz R: C5a receptor mediates neutrophil activation and ANCA-induced glomerulonephritis. J Am Soc Nephrol 2009;20:289-298.

7 Xiao H, Dairaghi DJ, Powers JP, Ertl LS, Baumgart T, Wang Y, Seitz LC, Penfold ME, Gan L, Hu P, Lu B, Gerard NP, Gerard C, Schall TJ, Jaen JC, Falk RJ, Jennette JC: C5a receptor (CD88) blockade protects against MPO-ANCA GN. J Am Soc Nephrol 2014;25:225-231.

8 Manenti L, Vaglio A, Gnappi E, Maggiore U, Allegri L, Allinovi M, Urban ML, Delsante M, Galetti M, Nicastro M, Pilato FP, Buzio C: Association of serum C3 concentration and histologic signs of thrombotic microangiopathy with outcomes among patients with ANCA-associated renal vasculitis. Clin J Am Soc Nephrol 2015;10:21432151.

$>9$ Berger BE: The alternative pathway of complement and the evolving clinical-pathophysiological spectrum of atypical hemolytic uremic syndrome. Am J Med Sci 2016;352:177-190.

10 Feng S, Eyler SJ, Zhang Y, Maga T, Nester CM, Kroll MH, Smith RJ, Afshar-Kharghan V: Partial ADAMTS13 deficiency in atypical hemolytic uremic syndrome. Blood 2013;122:1487-1493.

$>11$ Roumenina LT, Loirat C, Dragon-Durey MA, Halbwachs-Mecarelli L, Sautes-Fridman C, Frémeaux-Bacchi V: Alternative complement pathway assessment in patients with atypical HUS. J Immunol Methods 2011;365: 8-26.

12 Loirat C, Frémeaux-Bacchi V: Atypical hemolytic uremic syndrome. Orphanet J Rare Dis 2011;6:60.

13 Joseph C, Gattineni J: Complement disorders and hemolytic uremic syndrome. Curr Opin Pediatr 2013;25: 209-215.

14 Józsi M, Tortajada A, Uzonyi B, Goicoechea de Jorge E, Rodríguez de Córdoba S: Factor H-related proteins determine complement-activating surfaces. Trends Immunol 2015;36:374-384.

$\rightarrow 15$ Meri S: Complement activation in diseases presenting with thrombotic microangiopathy. Eur J Intern Med 2013;24:496-502.

16 Noris M, Remuzzi G. Atypical hemolytic-uremic syndrome. N Engl J Med 2009;361:1676-1687. 
17 Noris M, Caprioli J, Bresin E, Mossali C, Pianetti G, Gamba S, Daina E, Fenili C, Castelletti F, Sorosina A, Piras R, Donadelli R, Maranta R, van der Meer I, Conway EM, Zipfel PF, Goodship TH, Remuzzi G: Relative role of genetic complement abnormalities in sporadic and familial aHUS and their impact on clinical phenotype. Clin J Am Soc Nephrol 2010;5:1844-1859.

18 Ariceta G, Besbas N, Johnson S, Karpman D, Landau D, Licht C, Loirat C, Pecoraro C, Taylor CM, Van de Kar N, Vandewalle J, Zimmerhackl LB; European Paediatric Study Group for HUS: Guideline for the investigation and initial therapy of diarrhea-negative hemolytic uremic syndrome. Pediatr Nephrol 2009;24:687-696.

19 Nürnberger J, Philipp T, Witzke O, Opazo Saez A, Vester U, Baba HA, Kribben A, Zimmerhackl LB, Janecke AR, Nagel M, Kirschfink M: Eculizumab for atypical hemolytic-uremic syndrome. N Engl J Med 2009;360:542-544.

20 Mache CJ,Acham-Roschitz B, Frémeaux-Bacchi V, Kirschfink M, Zipfel PF, Roedl S, Vester U, Ring E: Complement inhibitor eculizumab in atypical hemolytic uremic syndrome. Clin J Am Soc Nephrol 2009;4:1312-1316.

21 Liu L, Li Y, Li S, Hu N, He Y, Pong R, Lin D, Lu L, Law M: Comparison of next-generation sequencing systems. J Biomed Biotechnol 2012;2012:251364.

$\checkmark 22$ Goodwin S, McPherson JD, McCombie WR: Coming of age: ten years of next-generation sequencing technologies. Nat Rev Genet 2016;17:333-351.

23 Chen M, Xing GQ, Yu F, Liu G, Zhao MH: Complement deposition in renal histopathology of patients with ANCAassociated pauci-immune glomerulonephritis. Nephrol Dial Transplant 2009;24:1247-1252.

24 Gou SJ, Yuan J, Wang C, Zhao MH, Chen M: Alternative complement pathway activation products in urine and kidneys of patients with ANCA-associated GN. Clin J Am Soc Nephrol 2013;8:1884-1891.

-25 Hilhorst M, van Paassen P, van Rie H, Bijnens N, Heerings-Rewinkel P, van Breda Vriesman P, Cohen Tervaert JW; Limburg Renal Registry: Complement in ANCA-associated glomerulonephritis. Nephrology, dialysis, transplantation: Official Publication of the European Dialysis and Transplant Association - European Renal Association 2015, Epub ahead of print.

26 Lyons PA, Rayner TF, Trivedi S, Holle JU, Watts RA, Jayne DR, Baslund B, Brenchley P, Bruchfeld A, Chaudhry AN, Cohen Tervaert JW, Deloukas P, Feighery C, Gross WL, Guillevin L, Gunnarsson I, Harper L, Hrušková Z, Little MA, Martorana D, Neumann T, Ohlsson S, Padmanabhan S, Pusey CD, Salama AD, Sanders JS, Savage CO, Segelmark M, Stegeman CA, Tesar V, Vaglio A, Wieczorek S, Wilde B, Zwerina J, Rees AJ, Clayton DG, Smith KG: Genetically distinct subsets within ANCA-associated vasculitis. N Engl J Med 2012;367:214-223.

27 Relle M, Föhr B, Fasola F, Schwarting A: Genetics and pathophysiology of granulomatosis with polyangiitis (GPA) and its main autoantigen proteinase 3. Mol Cell Probes 2016;30:366-373.

28 Xie G, Roshandel D, Sherva R, Monach PA, Lu EY, Kung T, Carrington K, Zhang SS, Pulit SL, Ripke S, Carette S, Dellaripa PF, Edberg JC, Hoffman GS, Khalidi N, Langford CA, Mahr AD, St Clair EW, Seo P, Specks U, Spiera RF, Stone JH, Ytterberg SR, Raychaudhuri S, de Bakker PI, Farrer LA, Amos CI, Merkel PA, Siminovitch KA: Association of granulomatosis with polyangiitis (Wegener's) with HLA-DPB1*04 and SEMA6A gene variants: evidence from genome-wide analysis. Arthritis Rheum 2013;65:2457-2468.

29 Bonatti F, Reina M, Neri TM, Martorana D: Genetic susceptibility to ANCA-associated vasculitis: state of the art. Front Immunol 2014;5:577.

-30 Rahmattulla C, Mooyaart AL, van Hooven D, Schoones JW, Bruijn JA, Dekkers OM; European Vasculitis Genetics Consortium, Bajema IM: Genetic variants in ANCA-associated vasculitis: a meta-analysis. Ann Rheum Dis 2016;75:1687-1692.

-31 Persson U, Truedsson L, Westman KW, Segelmark M: C3 and C4 allotypes in anti-neutrophil cytoplasmic autoantibody (ANCA)-positive vasculitis. Clin Exp Immunol 1999;116:379-382.

-32 Takahashi K, Tanabe K, Ohnuki M, Narita M, Ichisaka T, Tomoda K, Yamanaka S: Induction of pluripotent stem cells from adult human fibroblasts by defined factors. Cell 2007;131:861-872.

-33 Hariharan K, Kurtz A, Schmidt-Ott KM: Assembling kidney tissues from cells: the long road from organoids to organs. Front Cell Dev Biol 2015;3:70.

34 Stachelscheid H, Seltmann S, Lekschas F, Fontaine JF, Mah N, Neves M, Andrade-Navarro MA, Leser U, Kurtz A: CellFinder: a cell data repository. Nucleic Acids Res 2014;42(database issue):D950-D958.

-35 El Amrani K, Stachelscheid H, Lekschas F, Kurtz A, Andrade-Navarro MA: MGFM: a novel tool for detection of tissue and cell specific marker genes from microarray gene expression data. BMC Genomics 2015;16:645. 